\title{
Short PR intervals and tachyarrhythmias in Fabry's disease
}

\author{
J. Efthimiou, J. McLelland and D.J. Betteridge \\ Department of Medicine, University College Hospital, London WC1E 6JJ, UK.
}

\begin{abstract}
Summary: Two brothers with Fabry's disease presenting with palpitations were found to have intermittent supraventricular tachycardias. Their electrocardiograms, when symptom-free, revealed short PR intervals consistent with ventricular pre-excitation. Treatment of one of the brothers with verapamil resulted in improvement of the palpitations and reduction in frequency of the tachycardia. Recurrent supraventricular tachycardia associated with ventricular pre-excitation has not previously been described in Fabry's disease. Evidence suggests that this complication may be due to glycolipid deposition in the conducting system around the atrioventricular node.
\end{abstract}

\section{Introduction}

Fabry's disease (angiokeratoma corporis diffusum) is an X-linked disorder of glycolipid metabolism result ing in deposition of ceramide trihexoside, particularly in the skin, kidneys and cardiovascular system. Cardiac manifestations are numerous and include rhythm disturbances, myocardial infarction, and congestive or rarely hypertrophic cardiomyopathy (Colucci et al., 1982).

We report on two brothers known to have Fabry's disease who had intermittent tachyarrhythmias with electrocardiograms demonstrating a short PR interval, consistent with ventricular pre-excitation.

\section{Case reports}

Case 1

A 49 year old man known to have Fabry's disease since the age of 7 years, presented with a year's history of intermittent palpitations associated with dizziness. His previous medical history included a brain stem stroke at the age of 30 years, from which he fully recovered, and an anterior myocardial infarction at the age of 31 years.

Examination revealed finger clubbing (present for 6 years) and the typical angiectases over the buttocks, genitalia and thighs. The heart rate was 90 per minute and regular and the blood pressure $120 / 80 \mathrm{~mm} \mathrm{Hg}$. The heart sounds were normal, and there was no

Correspondence: J. Efthimiou B.Sc., M.R.C.P. (UK). Accepted: 7 August 1985 evidence of heart failure.

The full blood count, erythrocyte sedimentation rate, plasma electrolytes, cardiac enzymes, thyroid function tests and chest radiograph were normal. The creatinine clearance was reduced at $51 \mathrm{ml} / \mathrm{min}$, but urine analysis was normal with no proteinuria. The electrocardiogram revealed a short PR interval $(0.10 \mathrm{~s})$ with no delta wave (Figure 1). Electrocardiograms recorded 12 and 5 years earlier revealed normal PR intervals of $0.16 \mathrm{~s}$ and $0.12 \mathrm{~s}$ respectively. Twenty-four hour ambulatory electrocardiography revealed intermittent episodes of supraventricular tachycardia with rates up to 220 per minute (Figure 2), which were often accompanied by dizziness. An M-mode echocardiogram was normal.

Treatment with verapamil resulted in reduction in

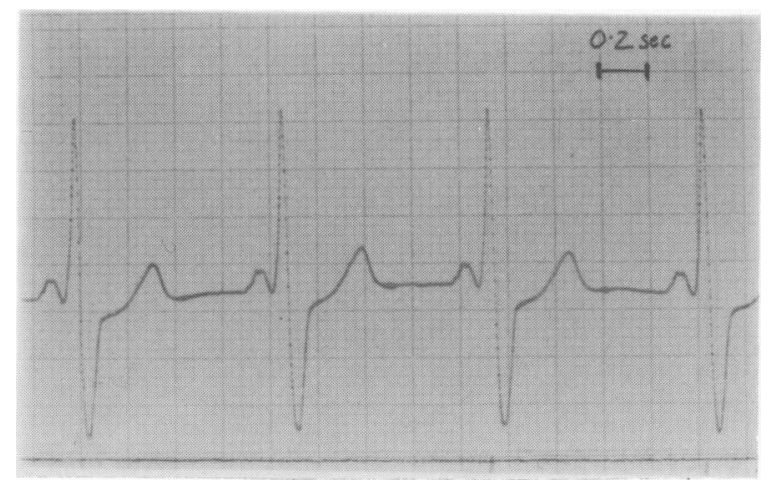

Figure 1 Electrocardiogram (standard lead II) of case 1 showing a short PR interval $(0.10 \mathrm{~s})$ with no delta wave.

C The Fellowship of Postgraduate Medicine, 1986 


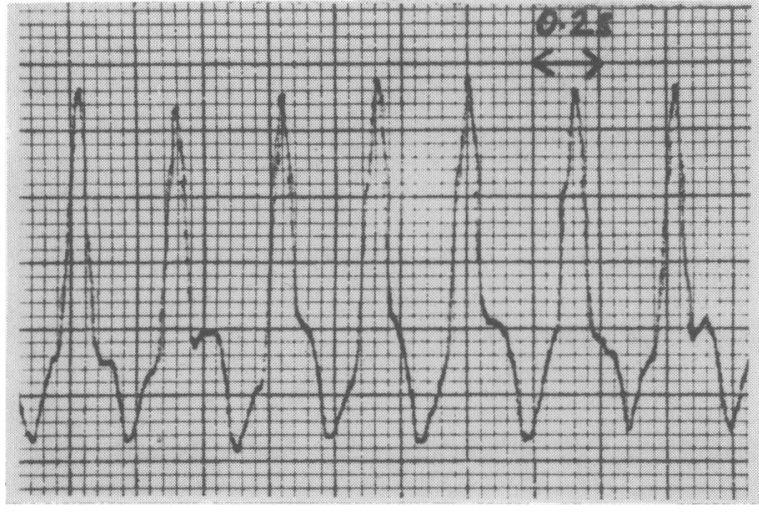

Figure 2 Twenty-four hour ambulatory electrocardiogram recording demonstrating a supraventricular tachycardia at a rate of 220 per minute (Case 1).

both the palpitations and dizziness, and further twenty-four hour ambulatory electrocardiography, whilst on treatment, revealed only one short episode of supraventricular tachycardia.

\section{Case 2}

The 45 year old brother of case 1 also known to have Fabry's disease since the age of 7 presented with a three month history of palpitations. Examination revealed clubbing of the fingers (present for 4 years) with the typical angiectases around the waist, genitalia and thighs. The heart rate was 78 per minute and regular and the blood pressure $115 / 70 \mathrm{~mm} \mathrm{Hg}$. The heart sounds were normal and there was no evidence of heart failure.

Routine haematology, biochemistry and chest radiography were normal, as in case 1 . The electrocardiogram, however, revealed a short PR interval

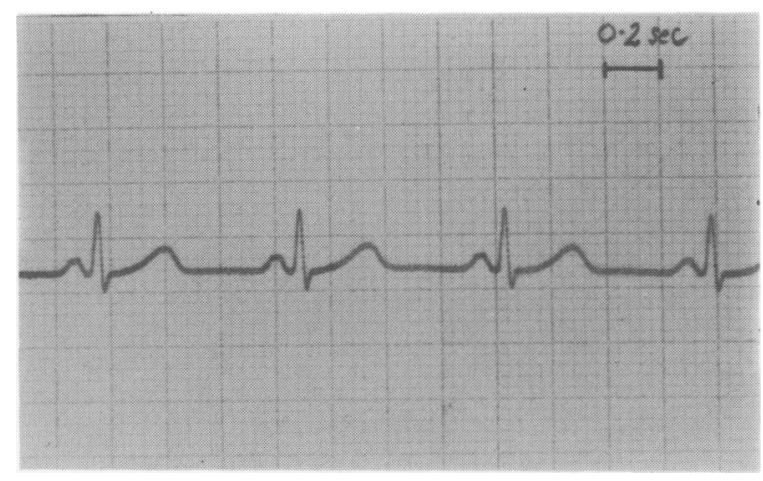

Figure 3 Electrocardiogram (standard lead II) of case 2 showing a short PR interval (0.11 s) with no delta wave.
(0.11 s) with a normal QRS complex (Figure 3). Twenty-four hour ambulatory electrocardiography demonstrated recurrent episodes of supraventricular tachycardia with rates up to 200 per minute. An Mmode echocardiogram was normal.

Treatment with anti-arrhythmic drugs was offered but declined.

A third brother with Fabry's disease died suddenly and unexpectedly at the age of 26 years following a run. The cause of death was thought to have been a tachyarrhythmia which was noted by a friend at the time of his collapse. Records of previous electrocardiograms were not available. Post-mortem studies revealed a normal sized heart with extensive glycolipid deposition in the conducting system around the atrioventricular node and bundle of His, with lesser infiltration elsewhere in the heart. There was no evidence of myocardial infarction or myocarditis.

\section{Discussion}

We have described two brothers known to have Fabry's disease with electrocardiograms demonstrating short PR intervals, with no delta waves, both of whom complained of palpitations and were found to have intermittent tachyarrhythmias. These features are consistent with the type of ventricular pre-excitation first described by Lown et al. (1952), in which the tachycardia, usually nodal re-entrant tachycardias, are the result of either circus movement involving the atrioventricular node and an atrioventricular nodal bypass, i.e. James bundle (James, 1961; Ward et al., 1978), or an intra-atrioventricular nodal abnormality (Caracta et al., 1973). The third brother who died suddenly at the age of 26 years may well have succumbed with a tachyarrhythmia related to ventricular pre-excitation, and post-mortem examination revealed extensive glycolipid deposition around his atrioventricular node and bundle of His. That the short PR interval is related to progressive glycolipid deposition in the conduction system is suggested by the finding of a progressively decreasing PR interval in case 1 over a 12 year period.

Cardiac involvement is not usually the predominant clinical manifestation of Fabry's disease, but it is present on pathological study in nearly all cases (Ferrans et al., 1969). Cardiovascular manifestations of Fabry's disease are numerous and include angina, myocardial infarction, rhythm and conduction disturbances, valvular (especially mitral) dysfunction, congestive or, rarely, hypertrophic cardiomyopathy and hypertension secondary to renal disease (Desnick \& Sweeley, 1983). Short PR intervals have only rarely been reported in Fabry's disease (Desnick \& Sweeley, 1983), and in 2 patients followed over 10 years the PR interval decreased progressively indicating accelerated 
atrioventricular conduction, with progressive glycolipid deposition in the bundle of His (Rowe \& Caralis, 1978). However, in these cases the patients did not present with palpitations, and the short PR interval was not associated with tachyarrhythmias.

Both of the patients described here demonstrated finger clubbing which has also not previously been reported in Fabry's disease and for which there was no obvious pulmonary, cardiac, or gastrointestinal cause. The finger clubbing may have been coincidental, although it is possible that glycolipid deposition around the nail beds was the cause, and it may be of relevance that enlargement of the distal interphalangeal joints has been previously reported in Fabry's disease (Caracta et al., 1973).

The two brothers presented here had well documented Fabry's disease with electrocardiogram demonstrating short PR intervals, in association with intermittent tachyarrhythmias. Ventricular pre-excitation

\section{References}

CARACTA, A.R., DAMATA, A.N., GALLAGHER, J.J., JOSEPHSON, M.E., VARGESE, P.J., LAU, S.H. \& WESTURA, E.E. (1973). Electrophysiological studies in the syndrome of short PR interval, normal QRS complex. American Journal of Cardiology, 31, 245.

COLUCCI, W.S., LORELL, B.H., SHOEN, F.J. WARHOL, M.J. \& GROSSMAN, W.C. (1982). Hypertrophic cardiomyopathy due to Fabry's disease. New England Journal of Medicine, 307, 926.

DESNICK, R.J. \& SWEELEY, C.C. (1983). Fabry's disease: galactosidase A deficiency. In The Metabolic Basis of Inherited Disease, Stanbury, J.B., Wyngaarden, J.B., Fredrickson, D.S., Goldstein, J.L. \& Brown, M.S. (eds), 5th ed. p. 906, McGraw-Hill: New York.

FERRANS, V.J., HIBBS, R.G. \& BURDA, C.D. (1969). The heart in Fabry's disease: a histochemical and electron microscopic study. American Journal of Cardiology, 24, 95. syndromes usually occur in an otherwise normal heart, but in Fabry's disease it seems likely to be related to glycolipid deposition around the atrioventricular node, as was demonstrated at post-mortem in the brother who died suddenly at the age of 26 years. It may be pertinent that another infiltrative cardiomyopathy, Pompe's disease (Type 2 glycogen storage disease), may also be associated with a short PR interval, and the cardiac abnormalities on light microscopy in the two diseases are strikingly similar (Keith et al., 1967). Early recognition of this complication in Fabry's disease and treatment with anti-arrhythmic drugs may reduce the morbidity and mortality associated with the disease.

\section{Acknowledgement}

We are grateful to Dr Kim for his valuable advice in preparing this report.

JAMES, T.N. (1961). Morphology of the human atrioventricular node with remarks pertinent to it's electrophysiology. American Heart Journal, 61, 756.

KEITH, J., ROWE, R. \& VLAD, P. (1967). Glycogen storage disease of the heart. In Heart Disease in Infancy and Childhood, Keith, J. (ed), 2nd ed. p. 1112. Macmillan: New York.

LOWN, B., GANONG, W.F. \& LEVINE, S.A. (1952). The syndrome of short $P R$ interval, normal $Q R S$ complex and paroxysmal rapid heart action. Circulation, 5, 693.

ROWE, J.W. \& CARALIS, D.G. (1978). Accelerated atrioventricular conduction in Fabry's disease: a case report. Angiology, 29, 562.

WARD, D.E., CAMM, A.J. \& SPURRELL, R.A. (1978). Reentrant tachycardia using two bypass tracts and excluding AV node, in short PR interval, normal QRS syndrome. British Heart Journal, 40, 1127. 


\section{Erratum}

Volume 62, No. 726, April 1986

'Short PR intervals and tachyarrhythmias in Fabry's disease' J. Efthimiou et al, pages 285-287

The acknowledgement should have read: We are grateful to Dr Kim Fox for his valuable advice in preparing this report. 